\title{
HLA-DQB1*06 is a risk marker for chlamydia reinfection in African American women
}

\author{
Kristin M Olson $\mathbb{D}^{1} \cdot$ Jianming Tang ${ }^{1} \cdot$ LaDraka' Brown $^{1} \cdot$ Christen G Press $^{1} \cdot$ William M Geisler ${ }^{1}$
}

Received: 8 November 2017 / Revised: 19 January 2018 / Accepted: 25 January 2018 / Published online: 26 February 2018

(c) Macmillan Publishers Limited, part of Springer Nature 2018

\begin{abstract}
Associations between human leukocyte antigen (HLA) variants and chlamydia-related outcomes have been inconsistent. We previously identified HLA-DQB $1 * 06$ as a risk marker for chlamydia reinfection in a cohort of predominately HIV-infected adolescents. As chlamydia reinfection can lead to reproductive complications, validation of this finding in HIV-seronegative women may help reveal the underlying biology. We performed HLA-DQB1 genotyping in HIV-seronegative, chlamydiainfected African American women who were evaluated for reinfection at 3- and 6-month visits after treatment. Of 185 evaluable women for whom $H L A-D Q B 1$ genotyping was performed, only HLA-DQB $1 * 06$ was associated with chlamydia reinfection $(P=0.009)$, with no evidence of a dose-response effect for this allele. African American women with HLADQB1*06 may warrant more frequent chlamydia screening. More comprehensive genotyping of HLA class II and neighboring genes is needed to establish whether HLA-DQB $1 * 06$ is a causal variant for chlamydia reinfection or a surrogate for other causal variants in the major histocompatibility complex.
\end{abstract}

\section{Introduction}

Chlamydia is the most prevalent sexually transmitted bacterial infection in the United States [1]. There is a major disparity in chlamydia rates by race, with African Americans having an almost sixfold higher rate than Caucasians. Limited studies suggest that untreated chlamydial infection may persist for a year or longer in up to $50 \%$ of chlamydiainfected women [2]. Recommended treatments for chlamydia are highly efficacious [3], but up to $20 \%$ of treated patients may experience reinfection within months of treatment [4]. Even in the absence of symptoms, both persistent chlamydial infection and reinfection may lead to severe sequelae, including pelvic inflammatory disease (PID) and tubal factor infertility (TFI) [5].

The underlying mechanism through which some women are more susceptible to chlamydia reinfection than others has not been fully elucidated. In gene knockout studies in mice, CD4+ T-helper type-1 responses have been shown to

William M Geisler

wgeisler@uabmc.edu

1 Department of Medicine, University of Alabama at Birmingham, Birmingham, AL, USA be necessary and sufficient for the clearance of chlamydia infection from the genital tract, whereas CD8 $+\mathrm{T}$ cells appear to have a lesser role in the development of protective immunity to chlamydia [6]. As such, human leukocyte antigen (HLA) association studies of chlamydia outcomes have predominately focused on HLA class II genes, although the results have in general been inconsistent [712]. In a few of those studies, HLA-DQB $1 * 06$ has been implicated as a risk marker for specific chlamydia outcomes [10-12]. In our previous studies of $H L A-D Q B 1$ alleles and chlamydia outcomes in a longitudinal cohort of mostly HIV-infected African American adolescents, we found that HLA-DQB $1 * 06$ was associated with chlamydia reinfection and possibly PID [11, 12]. The same allele group has also been reported by Kinnunen et al. [10] as a risk marker for chlamydia-related TFI.

As our previous study cohort was a predominately HIVinfected population, it was unknown whether the findings on HLA associations with chlamydia outcomes from that cohort could be generalized to HIV-seronegative African American women, the population with the most reported cases of chlamydia [1]. Therefore, we conducted a followup study in a cohort of HIV-seronegative African American women with uncomplicated chlamydia to confirm the association of HLA-DQB $1 * 06$ with chlamydia reinfection. 
Table 1 Baseline characteristics of chlamydia-infected African American participants $(n=185)$

\begin{tabular}{llll}
\hline Characteristic & $\begin{array}{l}\text { Reinfection }(n=38) n \\
(\%)\end{array}$ & $\begin{array}{l}\text { No reinfection }(n=147) \\
n(\%)\end{array}$ \\
\hline $\begin{array}{l}\text { Age, years, median (range) } \\
\text { Number of sexual partners last 3 months, }\end{array}$ & $22(16-32)$ & $22(16-38)$ \\
$\begin{array}{l}\text { median (range) } \\
\text { Hormonal contraceptive use, } n(\%)\end{array}$ & $1.5(0-5)$ & $1(0-9)$ & $0.284^{\mathrm{b}}$ \\
$\begin{array}{l}\text { Prior chlamydia, } n(\%) \\
\text { Concomitant infections at baseline, n (\%) }\end{array}$ & $20(52.6)$ & $67(45.6)$ & $70(47.6)$ \\
Trichomoniasis & $24(63.2)$ & & $0.167^{\mathrm{b}}$ \\
Candidiasis & $2(5.3)$ & $7(4.8)$ & $0.414^{\mathrm{c}}$ \\
Bacterial vaginosis & $3(7.9)$ & $21(14.3)$ & $37(25.2)$ \\
\hline
\end{tabular}

${ }^{\text {a }}$ Significance set at the $\alpha=0.05$ level

${ }^{\mathrm{b}}$ Wilcoxon's rank-sum test

'Pearson's $\chi^{2}$-test for independence

${ }^{\mathrm{d}}$ Fisher's exact test

\section{Results and discussion}

We performed high-resolution $H L A-D Q B 1$ genotyping (to four-digit specificity) using genomic DNA extracted from 185 HIV-seronegative African American women with uncomplicated chlamydia, who were evaluated for chlamydia reinfection at 3- and 6-month follow-up visits after treatment as part of a prospective study. Select participant characteristics based on demographical and clinical data collected at baseline (i.e., enrollment) are shown in Table 1. The median age was 22 years, $51 \%$ were asymptomatic, $26 \%$ had concomitant bacterial vaginosis, and $51 \%$ had prior chlamydia based on self-report and medical record review of laboratory test results. Chlamydia reinfection, defined as a positive Chlamydia trachomatis nucleic acid amplification test (NAAT) at the 3- and/or 6-month followup visit, occurred in 38 (20\%) participants. There was a trend $(P=0.095)$ toward a lower chlamydia reinfection rate in participants with prior chlamydia, but no other demographic or clinical characteristics were associated with chlamydia reinfection.

Of $H L A-D Q B 1$ allele groups analyzed at two-digit specificities, only DQB $1 * 06$ was associated with chlamydia reinfection (Table 2), with the odds of reinfection 2.71 times greater in those with HLA-DQB1*06 (95\% confidence interval $(\mathrm{CI})=1.31-5.86, P=0.009)$. At least one copy of DQB $1 * 06$ was present in $46 \%(n=86)$ of the study cohort. The association remained consistent after controlling for prior chlamydia in a multivariable regression model (adjusted $P=0.010$ ). At the four-digit resolution level, the most common alleles, DQB $1 * 0602$ and DQB $1 * 0604$, accounted for most of the DQB $1 * 06$ association with reinfection (Table 3). At least one copy of either DQB $1 * 0602$ or DQB $1 * 0604$ was present in $39 \%(n=72)$ of the study cohort. These allele associations were not statistically significant; however, the analysis was limited by the small sample size at the four-digit resolution level. Moreover, the finding of an unfavorable chlamydia outcome associated with DQB $1 * 0602$ was consistent with our previous finding from our predominately HIV-infected cohort $[11,12]$. We did not find a dose-response effect when evaluating for an association of number of HLA-DQB $1 * 06$ allele copies with chlamydia reinfection (Table 4).

The consistent association of HLA-DQB $1 * 06$ with chlamydia reinfection in women regardless of HIV infection status suggests that this allele group could serve as a risk marker for reinfection. Based on known associations with PID and TFI [10], DQB $1 * 06$ could also serve as a risk marker for reproductive complications after chlamydia infection. The implication of this risk association is that the HLA-DQB $1 * 06$ allele may impair protective immune responses that influence susceptibility to chlamydial infection and/or the ability to clear infection. HLA-DQB $1 * 06$ allele variants and/or associated haplotypes have been found to be associated with autoimmune diseases (multiple sclerosis and pemphigoid) and other infections (HIV and streptococcal infections) [13]. One of most widely reported is the HLA-DRB1*1501-DQB1*0602 haplotype association with multiple sclerosis. The next step in our investigation of immunogenetic determinants of $\mathrm{CT}$ reinfection is to comprehensively sequence the major histocompatibility complex (MHC) region in our study cohort, in order to assess for haplotype associations and to evaluate variants that are in linkage disequilibrium with the HLA-DQB $1 * 06$ allele.

Despite a limited sample size, this candidate gene approach was expected to be informative based our previously published results of an association between HLADQB $1 * 06$ and chlamydia reinfection, and based on inclusion of a study population with similar HLA-DQB $1 * 06$ 
Table 2 Analyses of $H L A-D Q B 1$ allele groups (two-digit specificities) and chlamydia reinfection

\begin{tabular}{|c|c|c|c|c|c|}
\hline DQB1 allele & Participant frequency $^{\mathrm{a}}(\mathrm{n}=185) n(\%)$ & Reinfection $(n=38) n(\%)$ & No reinfection $(n=147) n(\%)$ & OR $(95 \% \mathrm{CI})$ & $P$-value ${ }^{\mathrm{b}}$ \\
\hline$D Q B 1 * 02$ & $56(30 \%)$ & $8(21 \%)$ & $48(33 \%)$ & $0.55(0.22-1.24)$ & 0.169 \\
\hline$D Q B 1 * 03$ & $72(39 \%)$ & $12(32 \%)$ & $60(41 \%)$ & $0.67(0.30-1.41)$ & 0.300 \\
\hline$D Q B 1 * 04$ & $24(13 \%)$ & $7(18 \%)$ & $17(12 \%)$ & $1.73(0.62-4.39)$ & 0.267 \\
\hline$D Q B 1 * 05$ & $75(41 \%)$ & $13(34 \%)$ & $62(42 \%)$ & $0.71(0.33-1.48)$ & 0.374 \\
\hline$D Q B 1 * 06$ & $86(46 \%)$ & $25(66 \%)$ & $61(42 \%)$ & $2.71(1.31-5.86)$ & 0.009 \\
\hline
\end{tabular}

${ }^{\mathrm{a}}$ Frequency refers to individuals who had at least one allele

${ }^{\mathrm{b}}$ After Bonferroni correction, threshold significance is set at $\alpha_{\text {adjusted }}=(0.05 / 5)=0.01$ level, logistic regression models. CI, confidence interval; $\mathrm{OR}$, odds ratio for chlamydia reinfection

frequency and chlamydia reinfection rates [12]. An additional limitation is that our study focused on African Americans, which reflects the population routinely evaluated at our study site, and did not evaluate haplotypes. It remains to be seen whether the risk conferred by DQB $1 * 06$ applies to other races/ethnicities. Nonetheless, a more comprehensive analysis of genes and single-nucleotide polymorphisms in the MHC class II region should help with fine-mapping efforts and will facilitate a haplotype analysis.

The potential clinical translation of our study findings is that African American women with HLA-DQB1*06 may need more frequent chlamydia screening to detect initial chlamydia and reinfection after treatment, in order to facilitate timely treatment and prevention of reproductive complications. Conversely, analyses of $C$. trachomatis epitopes restricted by non-DQB $1 * 06$ alleles could identify correlates of immune protection and advance chlamydia vaccine development.

\section{Materials and methods}

\section{Study cohort and clinical procedures}

Women $\geq 16$ years of age presenting to the Jefferson County Department of Health Sexually Transmitted Diseases Clinic in Birmingham, Alabama, for treatment of a recent positive screening $C$. trachomatis NAAT were enrolled in a chlamydia immunogenetics study after providing written informed consent. Those who were pregnant, had a prior hysterectomy, were co-infected with HIV, syphilis, or gonorrhea, or had received antibiotics with antichlamydial activity in the prior 30 days were excluded. At enrollment, participants were interviewed regarding their demographics, symptoms, sexual history, and hormonal contraception use, and provided blood for genomic DNA. A pelvic examination was performed to obtain a vaginal swab specimen for wet mount testing to diagnose trichomoniasis, bacterial vaginosis, and candidiasis, and an endocervical swab specimen for chlamydia and gonorrhea testing by
NAAT (Aptima Combo 2 [AC2]; Hologic, Marlborough, MA) as per the manufacturer's instructions. Participants received azithromycin $1 \mathrm{~g}$ orally for chlamydia treatment and then returned for 3- and 6-month follow-up visits for repeat chlamydia testing by NAAT (AC2) to evaluate for chlamydia reinfection. Our analyses focused on women with confirmed chlamydia at enrollment. Women were classified as having chlamydia reinfection if they had a positive NAAT result for $C$. trachomatis at the 3- and/or 6month follow-up visit, as well as for any positive $C$. trachomatis NAAT result during any non-study visit within the 6-month study period (and at least 28 days since treatment). The study was approved by the University of Alabama at Birmingham Institutional Review Board and Jefferson County Department of Health.

\section{HLA genotyping}

Using genomic DNA extracted from peripheral blood (Gentra Puregen Kit, Qiagen Inc., Germantown, MD), we performed high-resolution $H L A-D Q B 1$ genotyping. Briefly, the $H L A-D Q B 1$ locus was amplified by gene-specific primers using polymerase chain reaction (PCR), then resolved by Sanger sequencing (Olerup, Inc., West Chester, PA) designed for capillary electrophoresis. Alleles were assigned using the ABI DNA Analyzer (Applied Biosystems, Foster City, CA). Individual alleles were resolved to four-digit specificities against a recent HLA database (Assign SBT v4.7, Olerup Inc.). Data analyses evaluated allele groups (i.e., 2-digit specificity) and four-digit alleles corresponding to distinct protein sequences.

\section{Statistical analysis}

Analyses of baseline participant characteristics and chlamydia reinfection were performed using the Wilcoxon's rank-sum, Pearson's $\chi^{2}$, or Fisher's exact tests as appropriate. Analyses of chlamydia reinfection by HLA allele frequency were performed using logistic regression or exact logistic regression as appropriate. Any participant characteristics or HLA alleles associated with chlamydia 
Table 3 Analyses of four-digit alleles in the $D Q B 1 * 06$ group and chlamydia reinfection

\begin{tabular}{|c|c|c|c|c|c|}
\hline Allelic variants & $\begin{array}{l}\text { Participant frequency }{ }^{\mathrm{a}}(n= \\
185) n(\%)\end{array}$ & $\begin{array}{l}\text { Reinfection }(n=38) n \\
(\%)\end{array}$ & $\begin{array}{l}\text { No reinfection }(n=147) n \\
(\%)\end{array}$ & OR $(95 \% \mathrm{CI})$ & $P$-value \\
\hline$D Q B 1 * 0602$ & $67(36 \%)$ & $18(47 \%)$ & $49(33 \%)$ & $1.80(0.87-3.71)$ & $0.111^{\mathrm{c}}$ \\
\hline$D Q B 1 * 0603$ & $12(6 \%)$ & $3(8 \%)$ & $9(6 \%)$ & $1.31(0.34-5.11)$ & $0.714^{\mathrm{d}}$ \\
\hline$D Q B 1 * 0604$ & $9(5 \%)$ & $4(11 \%)$ & $5(3 \%)$ & $3.34(0.85-13.11)$ & $0.088^{\mathrm{d}}$ \\
\hline$D Q B 1 * 0609$ & $7(4 \%)$ & $2(5 \%)$ & $5(3 \%)$ & $1.58(0.29-8.47)$ & $0.634^{\mathrm{d}}$ \\
\hline$D Q B 1 * 0602$ and $* 0604$ & $72(39 \%)$ & $20(53 \%)$ & $52(35 \%)$ & $2.03(0.99-4.21)$ & $0.054^{\mathrm{c}}$ \\
\hline
\end{tabular}

${ }^{\mathrm{a}}$ Frequency refers to individuals who had at least one allele

${ }^{\mathrm{b}}$ After Bonferroni correction, threshold significance set at the $\alpha_{\text {adjusted }}=(0.05 / 5)=0.01$ level

${ }^{\mathrm{c}}$ Logistic regression

${ }^{\mathrm{d} E x a c t}$ logistic regression. CI, confidence interval; OR, odds ratio for chlamydia reinfection.

Table 4 Analyses of $H L A-D Q B 1$ allele copy numbers and chlamydia reinfection

\begin{tabular}{lllll}
\hline DQB1* allele & $\begin{array}{l}\text { Participant } \\
\text { frequency } \\
(n=185)\end{array}$ & $\begin{array}{l}\text { Reinfection } \\
(n=38)\end{array}$ & $\begin{array}{l}\text { No } \\
\text { reinfection } \\
(n=147)\end{array}$ & $P$-value \\
\hline$D Q B 1 * 06$ & & & & $0.066^{\mathrm{b}}$ \\
0 Copies & $100(54 \%)$ & $14(37 \%)$ & $86(59 \%)$ & \\
1 Copy & $63(34 \%)$ & $19(50 \%)$ & $44(30 \%)$ & \\
2 Copies & $22(12 \%)$ & $5(13 \%)$ & $17(12 \%)$ & \\
$D Q B 1 * 0602$ & & & & $0.114^{\mathrm{b}}$ \\
and *0604 & & & & \\
0 Copies & $113(61 \%)$ & $18(47 \%)$ & $95(65 \%)$ & \\
1 Copy & $55(30 \%)$ & $16(42 \%)$ & $39(27 \%)$ & \\
2 Copies & $17(9 \%)$ & $4(11 \%)$ & $13(9 \%)$ & \\
\hline
\end{tabular}

${ }^{\text {a Significance set at the } \alpha=0.05 \text { level; }{ }^{\mathrm{b}} \text { Cochran-Armitage test for }}$ trend

reinfection on univariate analyses with a nominal $P$-value $<$ 0.1 were further evaluated using a multivariable logistic regression model. Analyses of chlamydia reinfection by HLA allele copy frequency were performed using the Cochran-Armitage test for trend. Statistical significance was set at the $\alpha=0.05$ level and a Bonferroni correction adjusted $\alpha$-level was used as appropriate. All statistical analyses were performed using SAS, version 9.4 (SAS Institute Inc., Cary, NC).

Acknowledgements We thank Hanne Harbison and Cynthia Poore for their assistance in collecting specimens and clinical data. We thank Hemant Tiwari for his review of this manuscript. This work was supported in part by the National Institute of Allergy and Infectious Diseases (R01AI09369 to W.M.G.) and by the National Center for Advancing Translational Sciences and the Eunice Kennedy Shriver National Institute of Child Health and Human Development (1TL1TR001418 and 1F31HD094539 to K.M.O.) of the National Institutes of Health. The content is solely the responsibility of the authors and does not necessarily represent the official views of the $U$. S. National Institutes of Health.

\section{Compliance with Ethical Standards}

Conflict of interest The authors declare that they have no conflict of interest.

\section{References}

1. Centers for Disease Control and Prevention. 2015 Sexually Transmitted Diseases Surveillance. 2016. Available at: https://www.cdc.gov/std/stats15/toc.htm (Accessed 23 October 2017).

2. Geisler WM. Duration of untreated, uncomplicated Chlamydia trachomatis genital infection and factors associated with chlamydia resolution: a review of human studies. J Infect Dis. 2010; 201 (Supplement_2): S104-S113.

3. Geisler WM, Uniyal A, Lee JY, Lensing SY, Johnson S, Perry $\mathrm{RC}$, et al. Azithromycin versus doxycycline for urogenital Chlamydia trachomatis infection. N Engl J Med. 2015; 373: 2512-21.

4. Hosenfeld CB, Workowski KA, Berman S, Zaidi A, Dyson J, Mosure D, et al. Repeat infection with chlamydia and gonorrhea among females: a systematic review of the literature. SexTransm Dis. 2009; 36: 478-89.

5. Haggerty CL, Gottlieb SL, Taylor BD, Low N, Xu F, Ness RB. Risk of sequelae after Chlamydia trachomatis genital infection in women. J Infect Dis. 2010; 201 (Suppl 2): S134-S155.

6. Morrison RP, Feilzer K, Tumas DB. Gene knockout mice establish a primary protective role for major histocompatibility complex class II-restricted responses in Chlamydia trachomatis genital tract infection. Infect Immun. 1995; 63: 4661-8.

7. Cohen CR, Sinei SS, Bukusi EA, Bwayo JJ, Holmes KK, Brunham RC. Human leukocyte antigen class II DQ alleles associated with Chlamydia trachomatis tubal infertility. Obst Gynecol. 2000; 95: 72-77.

8. Cohen CRM, Gichui JM, Rukaria RM, Sinei SSM, Gaur LK, Brunham RC. Immunogenetic correlates for Chlamydia trachomatis-associated tubal infertility. Obstet Gynecol. 2003; 101: 438-44.

9. Ness RBM, Brunham RC, Shen C, Bass DCM. Investigators FTPECHPS. Associations among human leukocyte antigen (HLA) class II DQ variants, bacterial sexually transmitted diseases, endometritis, and fertility among women with clinical pelvic inflammatory disease. Sex Transm Dis. 2004; 31: 301-4.

10. Kinnunen AH, Surcel H-M, Lehtinen M, Karhukorpi J, Tiitinen A, Halttunen M, et al. HLA DQ alleles and interleukin-10 polymorphism associated with Chlamydia trachomatis-related tubal 
factor infertility: a case-control study. Human Reprod. 2002; 17 : 2073-8.

11. Geisler WM, Tang J, Wang C, Wilson CM, Kaslow RA. Epidemiological and genetic correlates of incident Chlamydia trachomatis infection in North American adolescents. J Infect Dis. 2004; 190: 1723-9.

12. Wang C, Tang J, Geisler WM, Crowley-Nowick PA, Wilson CM, Kaslow RA. Human leukocyte antigen and cytokine gene variants as predictors of recurrent Chlamydia trachomatis infection in high-risk adolescents. J Infect Dis. 2005; 191: 1084-92.

13. Online Mendelian Inheritance in Man (Johns Hopkins University, Baltimore, MD, 2017). MIM Number: 604305. Last edited 6 April 2015, https://omim.org/entry/604305 (Accessed 23 October 2017). 